\title{
Влияние параметров кристаллической подложки на максимальную мощность кремниевых гетеропереходных солнечных элементов
}

\author{
() И.Е. Панайотти \\ Физико-технический институт им. А.Ф. Иофффе РАН, Санкт-Петербург, Россия \\ E-mail: panaiotti@mail.ioffe.ru
}

Поступило в Редакцию 18 июня 2021 г.

В окончательной редакции 13 сентября 2021 г.

Принято к публикации 14 сентября 2021 г.

\begin{abstract}
Исследовано влияние концентрации донорной примеси и времени жизни носителей заряда в кристаллической кремниевой подложке на максимальную мощность гетеропереходных тонкопленочных солнечных элементов. Использованная в расчетах модель учитывает особенности генерации фототока в условиях среднего или высокого уровней инжекции носителей заряда при произвольном соотношении между диффузионной длиной и толщиной полупроводниковой пластины. Предложенная методика позволяет с достаточной для практических целей точностью вычислять допустимые пределы вариаций параметров подложки, обеспечивающие заданные значения рабочих характеристик фотоэлектрических преобразователей.
\end{abstract}

Ключевые слова: гетеропереходные солнечные элементы, кристаллические кремниевые подложки, оптимальные параметры, максимальная мощность.

DOI: 10.21883/PJTF.2021.24.51793.18929

Интенсивное развитие солнечной энергетики диктует необходимость непрерывного совершенствования конструкций фотоэлектрических преобразователей. Одним из наиболее перспективных технологических решений для производства солнечных батарей является использование гетеропереходных тонкопленочных структур с кристаллическими кремниевыми подложками. Эффективность лучших образцов таких фотоэлектрических элементов достигает $26.7 \%$ [1].

Для производства высокоэффективных гетеропереходных тонкопленочных солнечных элементов - НІТ-элементов (HIT - heterojunction with intrinsic thin-layer solar cells) - обычно используют кристаллические подложки $c$-Si $n$-типа. Такой параметр, как исходное (равновесное) объемное время жизни свободных носителей заряда $\tau_{0}$, является показателем качества пластины (n)c-Si. В подложках современных НІТ-элементов $\tau_{0}$ составляет от $1.5-8.0 \mathrm{~ms}$ при концентрации доноров $N_{d} \geqslant 10^{15} \mathrm{~cm}^{-3}$. Толщина подложки $d$ может меняться в пределах 90-170 $\mu \mathrm{m}$. Экспериментально установлено, что величина этого параметра слабо влияет на рабочие характеристики НІТ-элементов [2,3]: при уменьшении $d$ на 40\% потери выходной мощности не превышают $5 \%$. В настоящее время при производстве фотоэлектрических преобразователей данного типа, как правило, используют подложки толщиной 150-170 $\mu \mathrm{m}$. Пленки гидрогенизированного аморфного кремния $\alpha$ - $\mathrm{Si}: \mathrm{H} p$ - и $n$-типа толщиной $15-20 \mathrm{~nm}$, выращенные поверх тонких $(\sim 5 \mathrm{~nm})$ буферных слоев с собственной проводимостью ( $i) \alpha$-Si : H, формируют на поверхностях пластины $(n) c-\mathrm{Si}$ гетеропереходы. Современные технологии пассивации позволяют эффективно подавлять поверхностные рекомбинационные процессы с помощью внедрения буферных слоев [4]. В высококачественных образцах НІТ-элемен- тов рекордные напряжения холостого хода составляют более $0.74 \mathrm{~V}$ [5]. Такие высокие показатели были достигнуты в том числе за счет существенного снижения суммарной скорости поверхностной рекомбинации (до десятых долей $\mathrm{cm} / \mathrm{s}$ ), так что результирующие потери оказались близки к уровням рекомбинационных процессов в объеме подложки $[4,6]$. Успехи современных технологий в обработке поверхностей кристаллического кремния сделали актуальной задачу более детального изучения возможности улучшения рабочих характеристик НІТ-элементов за счет оптимального выбора $\tau_{0}$ и $N_{d}$.

Целью настоящей работы является теоретическое исследование корреляции между максимальной мощностью НІТ-элементов и объемными параметрами пластин $(n) c-\mathrm{Si}$.

Метод оптимизации структуры НІТ-элементов, предложенный в [7], основывается на анализе рекомбинационных процессов [8] и не учитывает диффузионный перенос зарядов внутри подложки. У модели, описанной в [6], такой недостаток отсутствует, так как она рассматривает совместное влияние рекомбинации и амбиполярной диффузии носителей заряда на распределения их концентраций в подложке с произвольными параметрами. При этом плотность тока короткого замыкания $J_{s c}$ используется в качестве произвольно задаваемого параметра. Метод не только позволяет моделировать процессы в НІТ-элементах при различных уровнях рекомбинационных потерь в объеме и на поверхностях кремниевых пластин, но и дает возможность рассмотреть раздельно объемные и поверхностные факторы, влияющие на вид вольт-амперных характеристик.

С целью решения поставленной задачи в настоящей работе рассматривается идеализированная полупровод- 


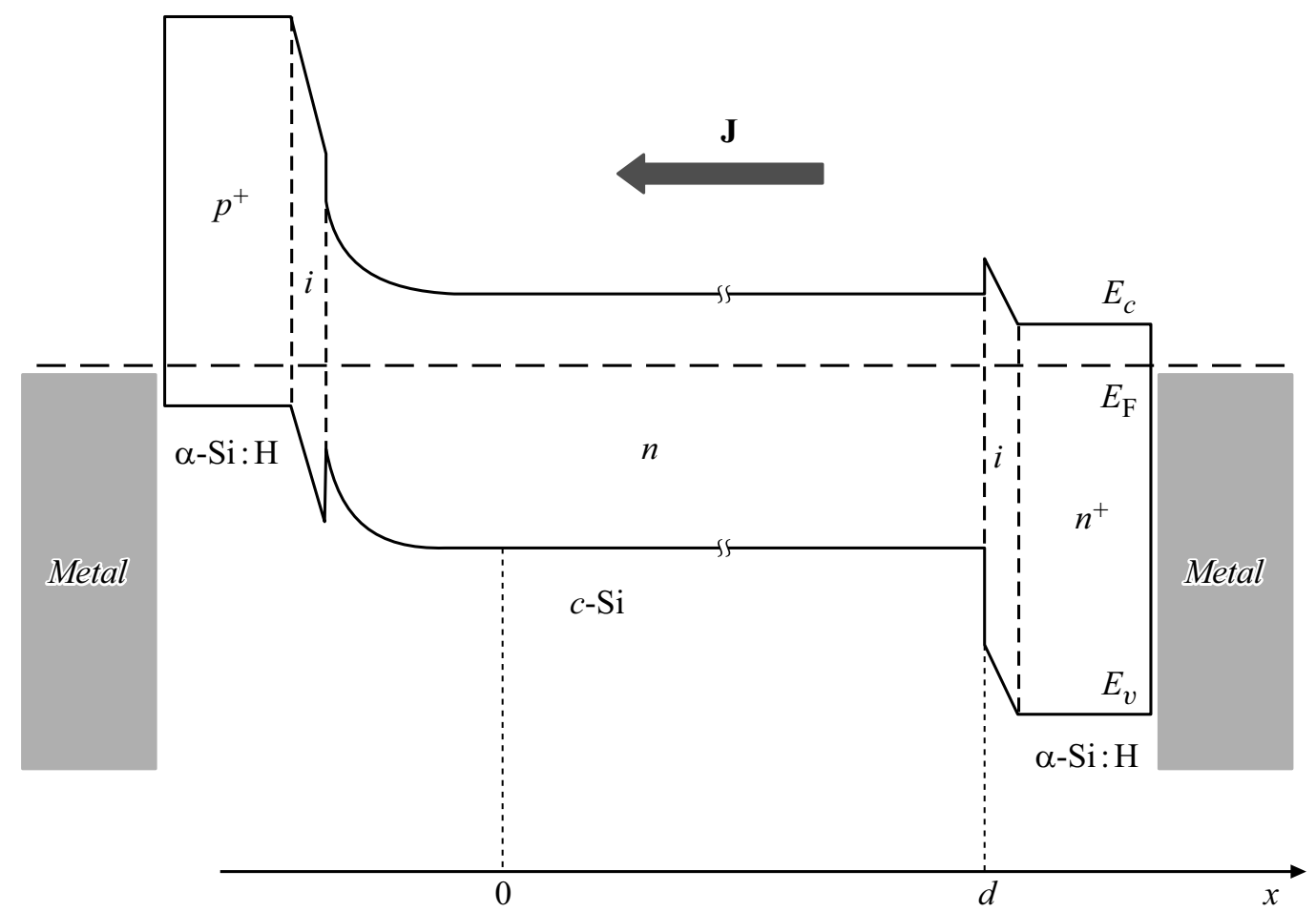

Рис. 1. Модель зонной диаграммы кристаллической подложки НІТ-элемента. $E_{c}, E_{v}-$ энергетические уровни дна зоны проводимости и потолка валентной зоны; $E_{\mathrm{F}}$ - энергетический уровень Ферми.

никовая структура без поверхностной рекомбинации. Расчеты проводились на основе теоретических зависимостей, полученных в приближении отсутствия загиба энергетических зон подложки со стороны $n^{+}-n$-контакта (рис. 1) [6]:

$$
\begin{gathered}
J=-J_{s c}+q \Delta p \sqrt{\frac{D}{\tau}} \tanh \left(\frac{d}{\sqrt{D \tau}}\right), \\
U=\frac{k T}{q} \ln \left\{\frac{\Delta p\left(\Delta p+N_{d}\right)}{n_{i}^{2}(T)}\right\} .
\end{gathered}
$$

Здесь $J<0$ - проекция на ось $x$ вектора плотности тока $\mathbf{J} ; U-$ величина прямого смещения $p^{+}-n$ гетероперехода; $\Delta p$ - концентрация избыточных носителей заряда на правой границе области пространственного заряда $p^{+}-n$-гетероперехода; $T-$ температура, равная $300 \mathrm{~K}$;

$$
D=D_{p} \frac{2 b \Delta p+b N_{d}}{\Delta p(b+1)+b N_{d}}
$$

- коэффициент амбиполярной диффузии [9]; $D_{p}-$ коэффициент диффузии дырок; $b=2.8$ - отношение коэффициентов диффузии электронов и дырок в $c-\mathrm{Si}$ при $T=300 \mathrm{~K}$;

$$
\tau=\left[\tau_{0}^{-1}+\tau_{\text {Auger }}^{-1}\right]^{-1}
$$

- результирующее объемное время жизни носителей заряда;

$$
\tau_{\text {Auger }}=\left[C_{n}\left(N_{d}+\Delta p\right)^{2}+C_{p}\left(N_{d}+\Delta p\right) \Delta p\right]^{-1}
$$

— время жизни носителей заряда при оже-рекомбинации;

$$
\begin{aligned}
& C_{n}=\left[2.8 \cdot 10^{-31}+\left(2.5 \cdot 10^{-22}\right) /\left(N_{d}+\Delta p\right)^{0.5}\right] \mathrm{cm}^{6} / \mathrm{s}, \\
& C_{p}=10^{-31} \mathrm{~cm}^{6} / \mathrm{s}[6]
\end{aligned}
$$

$n_{i}(T)$ - собственная равновесная концентрация носителей заряда в подложке при заданной температуре $300 \mathrm{~K} ; k$ - постоянная Больцмана; $q$ - элементарный заряд. Выражения (1) и (2) представляют собой вольтамперные характеристики НІТ-элемента. Если из (2) найти

$$
\Delta p(U)=-\frac{N_{d}}{2}+\sqrt{\frac{N_{d}^{2}}{4}+n_{i}^{2}(T) \exp \left(\frac{q U}{k T}\right)}
$$

и подставить в (1), то получится зависимость $J(U)$. Падение напряжения на НІТ-элементе в основном определяется величиной прямого смещения $p^{+}-n$-гетероперехода [8]. Последовательное сопротивление, связанное с падениями напряжения на других слоях структуры, в модели не учитывается. Плотность максимальной 


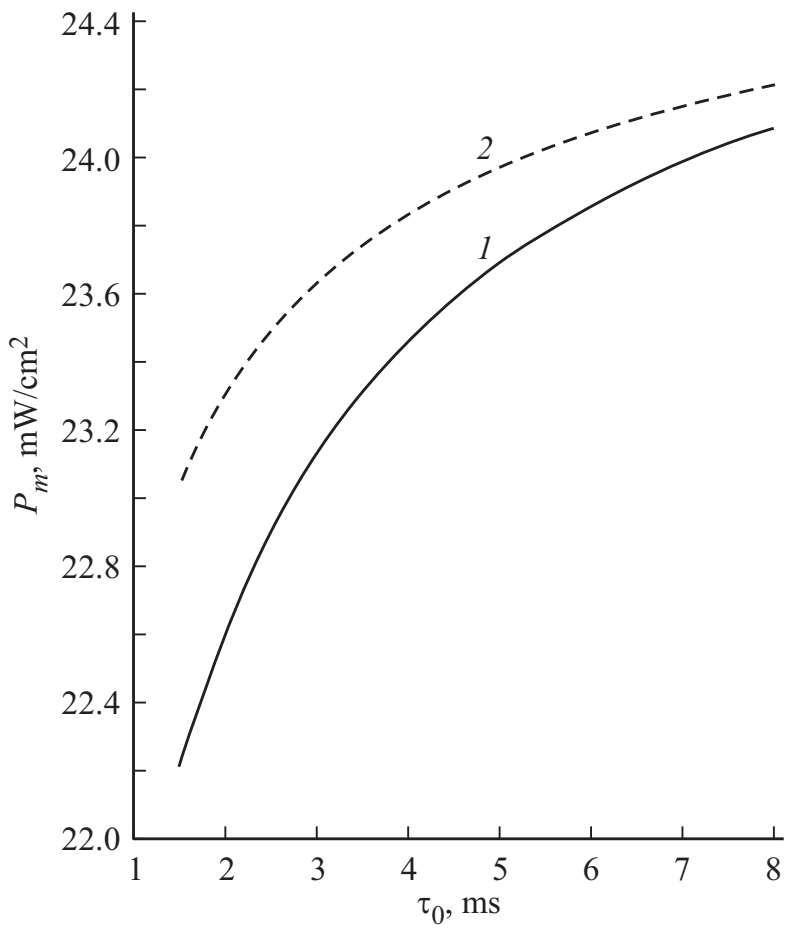

Рис. 2. Зависимость плотности максимальной мощности от величины исходного времени жизни носителей заряда в подложке НІТ-элемента. $J_{s c}=39 \mathrm{~mA} / \mathrm{cm}^{2}, d=160 \mu \mathrm{m} . N_{d}=10^{15}(1)$ и $5 \cdot 10^{15} \mathrm{~cm}^{-3}(2)$.

Данные промежуточных вычислений к рис. 2 для границ диапазона $\tau_{0}$

\begin{tabular}{c|c|c|c|c|c}
\hline $\begin{array}{c}P_{m}, \\
\mathrm{~mW} / \mathrm{cm}^{2}\end{array}$ & $\begin{array}{c}N_{d}, \\
\mathrm{~cm}^{-3}\end{array}$ & $\begin{array}{c}\tau_{0}, \\
\mathrm{~ms}\end{array}$ & $\begin{array}{c}\Delta p \\
\mathrm{~cm}^{-3}\end{array}$ & $\begin{array}{c}\tau, \\
\mathrm{ms}\end{array}$ & $\begin{array}{c}D \\
\mathrm{~cm}^{2} / \mathrm{s}\end{array}$ \\
\hline 22.22 & $10^{15}$ & 1.5 & $1.33 \cdot 10^{15}$ & 1.44 & 15.21 \\
23.06 & $5 \cdot 10^{15}$ & 1.5 & $8.59 \cdot 10^{14}$ & 1.27 & 12.70 \\
24.09 & $10^{15}$ & 8.0 & $3.42 \cdot 10^{15}$ & 4.87 & 16.20 \\
24.21 & $5 \cdot 10^{15}$ & 8.0 & $2.08 \cdot 10^{15}$ & 3.46 & 13.65
\end{tabular}

мощности $P_{m}$ можно численно рассчитать с помощью выражений (1) и (2) из условия $d P / d U=0$, где $P=J U$. В расчетах теоретических кривых (рис. 2) были использованы типичные значения $d, N_{d}$ и $J_{s c}$. Выбранная в качестве произвольного параметра плотность тока короткого замыкания, равная $39 \mathrm{~mA} / \mathrm{cm}^{2}$, характерна для современных НІТ-элементов, имеющих эффективность $\sim 20-22 \%$ при эталонной солнечной радиации $P_{\text {in }}=100 \mathrm{~mW} / \mathrm{cm}^{2}[2,10]$.

Преобразование солнечной энергии сопровождается интенсивным накоплением избыточных носителей заряда внутри подложки, причем их концентрации напрямую зависят от скорости рекомбинационных процессов. В режиме максимальной мощности $\Delta p$ становятся сравнимыми или выше концентрации донорной примеси [6]. При этом вследствие влияния процессов ожерекомбинации результирующее время жизни электронов и дырок $\tau$ оказывается намного меньше $\tau_{0}$ [11]. Кроме того, наблюдается существенный рост коэффициента амбиполярной диффузии носителей заряда относительно величины $D_{p}$. Согласно выражениям (3)-(5), чем выше $\Delta p$, тем слабее $D$ и $\tau$ зависят от уровня легирования кристаллической подложки. Поэтому по мере увеличения $\tau_{0}$ происходит как уменьшение разности между плотностями максимальной мощности у НІТ-элементов, изготовленных на низкоомных $\left(N_{d}=5 \cdot 10^{15} \mathrm{~cm}^{-3}\right)$ и высокоомных $\left(N_{d}=10^{15} \mathrm{~cm}^{-3}\right)$ подложках, так и существенное замедление роста функции $P_{m}\left(\tau_{0}\right)$ (рис. 2). Таблица содержит данные промежуточных вычислений к рис. 2 для границ диапазона $\tau_{0}$. Таким образом, при использовании пластин $c$-Si c повышенной концентрацией донорной примеси выигрыш в величине максимальной мощности оказывается заметным только при сравнительно небольших значениях исходных времен жизни носителей заряда.

Теоретические кривые плотности максимального тока $J_{m}$ в целом повторяют ход зависимостей $P_{m}\left(\tau_{0}\right)$ и также сближаются по мере увеличения исходного времени жизни носителей заряда (рис. 3,a). Однако

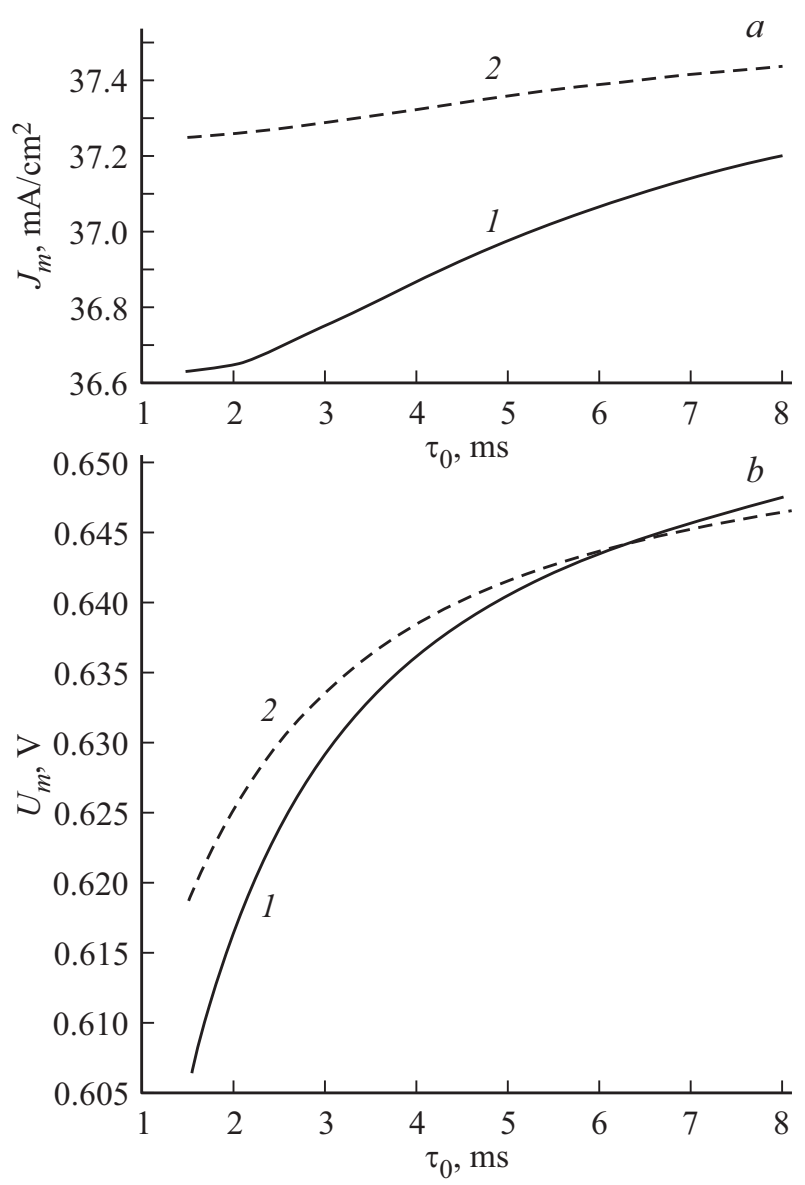

Рис. 3. Зависимости плотности максимального тока $(a)$ и максимального падения напряжения на $p^{+}-n$-гетеропереходе $(b)$ от величины исходного времени жизни носителей заряда в подложке НІТ-элемента. $J_{s c}=39 \mathrm{~mA} / \mathrm{cm}^{2}, d=160 \mu \mathrm{m}$. $N_{d}=10^{15}(1)$ и $5 \cdot 10^{15} \mathrm{~cm}^{-3}(2)$. 
при $N_{d}=5 \cdot 10^{15} \mathrm{~cm}^{-3}$ функция $J_{m}\left(\tau_{0}\right)$ растет заметно медленнее, чем при $N_{d}=10^{15} \mathrm{~cm}^{-3}$. На рис. $3, b$ представлены результаты расчетов максимального напряжения $U_{m}$. Вид графиков функций $U_{m}\left(\tau_{0}\right)$ свидетельствует о том, что преимущество использования низкоомных подложек с целью повышения максимального напряжения исчезает, если исходное время жизни носителей заряда в пластине $(n) c-\mathrm{Si}$ превосходит $6.48 \mathrm{~ms}$. При $\tau_{0}>6.48 \mathrm{~ms}$ рекомбинационные потери таковы, что, как следует из выражения (2), величина произведения $\Delta p\left(\Delta p+N_{d}\right)$ оказывается больше в высокоомных подложках, чем в низкоомных. Согласно оценкам, времена жизни и концентрации носителей заряда в точке пересечения кривых $U_{m}\left(\tau_{0}\right)$ на рис. $3, b$ соответственно составляют $\tau \approx 4.35 \mathrm{~ms}, \Delta p \approx 3.23 \cdot 10^{15} \mathrm{~cm}^{-3}$ при $N_{d}=10^{15} \mathrm{~cm}^{-3}$ и $\tau \approx 3.17 \mathrm{~ms}, \Delta p \approx 1.96 \cdot 10^{15} \mathrm{~cm}^{-3}$ при $N_{d}=5 \cdot 10^{15} \mathrm{~cm}^{-3}$.

Полученные результаты могут быть использованы в исследовательских проектах, направленных на поиск оптимальных параметров подложек для НІТ-элементов.

\section{Финансирование работы}

Работа выполнена в рамках государственного задания ФТИ им. А.Ф. Иоффе в области фундаментальных научных исследований.

\section{Конфликт интересов}

Автор заявляет, что у нее нет конфликта интересов.

\section{Список литературы}

[1] K. Yoshikawa, H. Kawasaki, W. Yoshida, T. Irie, K. Konishi, K. Nakano, T. Uto, D. Adachi, M. Kanematsu, H. Uzu, K. Yamamoto, Nature Energy, 2, 17032 (2017). DOI: $10.1038 /$ nenergy.2017.32

[2] D. Andronikov, A. Abramov, S. Abolmasov, K. Emtsev, G. Ivanov, I. Nyapshaev, D. Orekhov, A. Semenov, G. Shelopin, E. Terukova, E. Terukov, N. Belkova, A. Dubrovskiy, P. Ishmuratov, A. Ivanov, D. Saykin, A. Smirnov, N. Saymurzanov, E. Sokolov, V. Tarasov, in Proc. 35th European Photovoltaic Solar Energy Conf. and Exhibition (EU PVSEC 2018), ed. by P. Verlinden, P. Helm, R. Kenny (Curran Associates, Inc., N.Y., 2019), vol. 1, p. 690. http://toc.proceedings.com/42395webtoc.pdf

[3] Е.И. Теруков, А.С. Абрамов, Д.А. Андронников, К.В. Емцев, И.Е. Панайотти, А.С. Титов, Г.Г. Шелопин, ФТП, 52 (7), 792 (2018). DOI: 10.21883/FTP.2018.07.46054.8781 [E.I. Terukov, A.S. Abramov, D.A. Andronikov, K.V. Emtsev, I.E. Panaiotti, A.S. Titov, G.G. Shelopin, Semiconductors, 52 (7), 931 (2018). DOI: 10.1134/S1063782618070230].

[4] J. Melskens, B.W.H. van de Loo, B. Macco, L.E. Black, S. Smit, W.M.M. Kessels, IEEE J. Photovolt., 8 (2), 373 (2018). DOI: 10.1109/JPHOTOV.2018.2797106
[5] A. Danel, S. Harrison, F. Gérenton, A. Moustafa, R. Varache, J. Veirman, C. Roux, in Proc. 35th European Photovoltaic Solar Energy Conference Conf. and Exhibition (EU PVSEC 2018), ed. by P. Verlinden, P. Helm, R. Kenny (Curran Associates, Inc., N.Y., 2019), vol. 1, p. 444. http://toc.proceedings.com/42395webtoc.pdf

[6] И.Е. Панайотти, Е.И. Теруков, И.С. Шахрай,

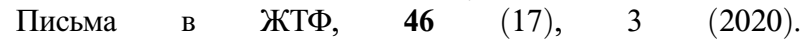
DOI: $10.21883 /$ PJTF.2020.17.49883.18377 [I.E. Panaiotti, E.I. Terukov, I.S. Shakhrai, Tech. Phys. Lett., 46 (9), 835 (2020). DOI: 10.1134/S1063785020090072].

[7] А.В. Саченко, Ю.В. Крюченко, В.П. Костылев, И.О. Соколовский, А.С. Абрамов, А.В. Бобыль, И.Е. Панайотти, Е.И. Теруков, ФТП, 50 (2), 259 (2016). [A.V. Sachenko, Yu.V. Kryuchenko, V.P. Kostylyov, I.O. Sokolovskyi, A.S. Abramov, A.V. Bobyl, I.E. Panaiotti, E.I. Terukov, Semiconductors, 50 (2), 257 (2016). DOI: $10.1134 / \mathrm{S} 1063782616020226]$.

[8] А.В. Саченко, А.И. Шкребтий, Р.М. Коркишко, В.П. Костылев, Н.Р. Кулиш, И.О. Соколовский, ФТП, 49 (2), 271 (2015). [A.V. Sachenko, A.I. Shkrebtii, R.M. Korkishko, V.P. Kostylyov, N.R. Kulish, I.O. Sokolovskyi, Semiconductors, 49 (2), 264 (2015). DOI: $10.1134 / \mathrm{S} 1063782615020189]$.

[9] К.В. Шалимова, Физика полупроводников (Энергоатомиздат, М., 1985), с. 249-266.

[10] A.S. Abramov, D.A. Andronikov, S.N. Abolmasov, E.I. Terukov, in High-efficient low-cost photovoltaics. Recent developments, 2nd ed. Springer Ser. in Optical Sciences (Springer Nature, Switzerland, 2020), vol. 140, ch. 7.

[11] E. Kobayashi, S. De Wolf, J. Levrat, G. Christmann, A. Descoeudres, S. Nicolay, M. Despeisse, Y. Watabe, C. Balif, Appl. Phys. Lett., 109 (15), 153503 (2016). DOI: $10.1063 / 1.4964835$ 